\title{
Nano-formulations: high definition liquid engineering of pesticides for advanced crop protection in agriculture
}

\section{Introduction}

With the development of nanotechnology application in numerous scientific disciplines, it is expected that nanoscale products have been and will be used for agricultural, vector, and urban pest control prior to a complete evaluation of exposure and risk. ${ }^{1}$ Significant differences may exist between nanotechnology-based formulation and conventional formulations, primarily due to size and surface characteristics. Recent advances in agricultural research have triggered great interest in the exploration of nanotechnology. Leveraging nanomaterials in plant delivery studies, e.g. carbon nanotubes (CNTs) andmetal/metaloxide nanoparticles (NPs), have been shown to improve seed germination and plant growth. ${ }^{2}$ Pesticide nanodelivery techniques, including nano-encapsu-lates, nanocages and nanocontainers, are more efficient and lead to less pesticide release into the environment and rapid degradation of the pesticide in the soil. ${ }^{3}$ The greener nanotechnology approach, with nanoemulsions containing natural oils, surfactants and water, is very promising and could be adopted pragmatically.

\section{Nanogel}

Nanogels may be defined as nano-sized hydrogel systems which are highly cross linked systems in nature involving polymer systems, which are either co-polymerized or monomeric. ${ }^{4}$ Sudden outbreak in the field of nanotechnology have introduced the need for developing nanogel systems which proven their potential to deliver active ingredients in controlled, sustained and targetable manner. With the emerging field of polymer sciences it has now become inevitable to prepare smart nano-systems which can prove effective for treatment as well as clinical trials progress. Nevertheless, these systems have been investigated from a longer period of time for mak-ing advancements in synthetic procedures not only for active delivery but for miscellaneous agents like quantum dots, dyes and other diagnostic agents. ${ }^{5-7}$ Traditionally in the name of gels we have heard of semisolid formulations with three dimensional network of organic systems encompassing fluids and drugs. Majorly these systems have been the part of traditional sys-tem of topical drug delivery for local effects. Prospects of targeted delivery perhaps could not been established with these preparations. ${ }^{8}$ The significance of nanosized microgel and hydrogel has arisen due to specific delivery system anticipation. Wide variety of polymer systems and the easy alteration of their physicochemical characteristics have given advantage for versatile form of nanogel formulations. ${ }^{9}$ Recent studies at clinical level have shown promising value of nanogels. ${ }^{10}$ Nanogels are typical formulations mainly of the size range of $100 \mathrm{~nm}$, by varying solvent quality and branching the volume fraction, one can alter variably to maintain a three dimensional structure. ${ }^{11}$ The review suggests that innovation in this field shall bring-forth sound support to pesticide applications in future. Recently Institute of Pesticide Formulation Technology, Gurgaon has developed a nanogel formulation of permethrin (a synthetic pyrethroid insecticide) for long lasting impregnation of this insecticide in the dresses which can protect personals from mosquito
Volume 6 Issue $3-2017$

\author{
Dipak Kumar Hazra \\ AINP on Pesticide Residue Laboratory, Department of \\ Agricultural Chemicals, India
}

Correspondence: Dipak Kumar Hazra, AINP on Pesticide Residue Laboratory, Department of Agricultural Chemicals, Bidhan Chandra Krishi Viswavidyalaya, Directorate of Research, Research Complex Building, Kalyani-74I 235, Nadia,West Bengal, India, Email dipakipft@gmail.com

Received: January 05, 2017 | Published: February 03, 2017

bite whenever they are deployed/posted to work in forest areas. ${ }^{12}$ The formulation developed in the laboratory was evaluated as per WHO specifications with respect to wash resistance and it qualifies all the laid down parameters. This type of nanogel formulation may have good future in seed dressing/coating because of its lower particle size, large surface area and greater adhesive properties.

\section{Nanoemulsions}

Nanoemulsions have a particle size of less than 200nm, which makes the systems inherently transparent/translucent and kinetically stable. Pesticides formulated with nanoemulsions having a lower surfactant concentration than microemulsions and surfactants are considerably more environmentally friendly and are cost effective and economically viable. ${ }^{13}$ Low-energy emulsification methods are applied to produce nanoemulsions, and the energy stored could promote smaller-sized nanoparticles of longer life. ${ }^{14-16}$ A 5\% nanoemulsion formulation of chlorpyriphos was prepared, physicchemically characterized and larvicidal activity was evaluated against the perpetually troublesome mosquitoes (Aedes aegypti) at Institute of Pesticide Formulation Technology, Gurgaon. ${ }^{13}$ Efficacy of commercially available emulsifiable concentrate was compared with nanoemulsion by performing larvicidal bioassay on late $3^{\text {rd }}$ and early 4th in-star larvae. The experiment was carried out at $26-29^{\circ} \mathrm{C}$ and relative humidity $65-70 \%$. All treatments were set up in triplicates and percentage mortalities were determined after 24hours of treatment. Mortality percentage ranged from 88.35-100.00 in nanoemulsion and 86.67-98.35 in EC, respectively. It can be predicted that at lower concentration (300-1500ppm), nanoemulsion can be very effective against mosquitoes due to smaller droplet size of nanopesticide with increased surface area and better surface coverage property.

\section{Acknowledgements}

None. 


\section{Conflict of interest}

The author declares no conflict of interest.

\section{References}

1. Kuzma J, VerHage P. Nanotechnology in agriculture and food production: Anticipated applications. 2010. 44 p.

2. Nair R, Varghese SH, Nair BG, et al. Nanoparticulate material delivery to plants. Plant Science. 2010;179(3):154-63.

3. Sadurni N, Solans C, Azemar N, et al. Studies on the formation of O/W nano-emulsions, by low-energy emulsification methods, suitable for pharmaceutical application. European Journal Pharmaceutical Science. $2005 ; 26(5): 438-451$.

4. Phillips MA, Gran ML, Peppas NA. Targeted nanodelivery of drugs and diagnostics. Nano Today. 2010;5(2):143-159.

5. Hasegawa U, Nomura SM, Kaul SC, et al. Nanogel quantum dots hybrid nanoparticles for live cell imaging. Biochemical and Bio-physical Research Communications. 2005;331(4):917-921.

6. Wu W, Aiello M, Zhou T, et al. In-situ immobilization of quantum dots in polysaccharide based nanogel for integration of optical $\mathrm{pH}$ sensing, tumor cell sensing and drug delivery. Biomaterials. 2010;31(11):30233031 .

7. Gong Y, Fan M, Gao F, et al. Preparation and characterization of amino functionalized magnetic nanogels via photopoly-merisation for MRI applications. Colloids and Surfaces B. 2009;71(2):243-247.

8. Sun H, Yu J, Gong P, et al. Novel core shell magnetic nanogels synthesized in emulsion free aqueous systems under UV irradiation for targeted radiopharmaceutical applications. Journal of Magnetism and Magnetic Materials. 2005;294(3):273-80.
9. Djordjevic J, Michniak B, Uhrich KE. Amphiphilic star like macromolecules as novel carriers for topical delivery of non steroidal anti-inflammatory drugs. AAPS Pharm Sci Tech. 2003;5(4):1-12.

10. Oishi M, Miyagawa N, Sakaru T, et al. pH-responsive nanogel containing platinum nanoparticles:Applications to on-off regulation of catalytic activity for reactive oxygen species. Reactive Functional Polymers. 2007;67(7):662-668.

11. Kageyama S, Kitano S, Hirayama M, et al. Humoral immune responses in patients vaccinated with 1-146 HER-2 protein complexed with cholesteryl pullulan nanogel. Cancer Science. 2008;99(3):601-607.

12. Mourey TH, Leon JW, Bennet JR, et al. Characterizing property distributions of polymeric nanogels by size-exclusion chromatography. Journal of Chromatography A. 2007;1146(1):51-60.

13. Hazra DK, Megha P, Raza SK, et al. Patanjali Formulation technology:key parameters for food safety with respect to agrochemicals use in crop protection. T Journal of Plant Protection Sciences. 2013;5(2):1-19.

14. Lim CJ, Basri M, Omar D, et al. Self-assembly behaviour of alkylpolyglucosides (APG) in mixed surfactant-stabilized emulsions system. Journal of Molecular Liquids. 2011;158(3):175-181.

15. Izquierdo P, Feng J, Esquena J, et al. The influence of surfactant mixing ratio on nano-emulsion formation and stability. Journal of Colloid and Interface Science. 2005;285(1):388-394.

16. Knowles A. Recent developments of safer formulations of agrochemicals. Environmentalist. 2008;28(1):35-44. 